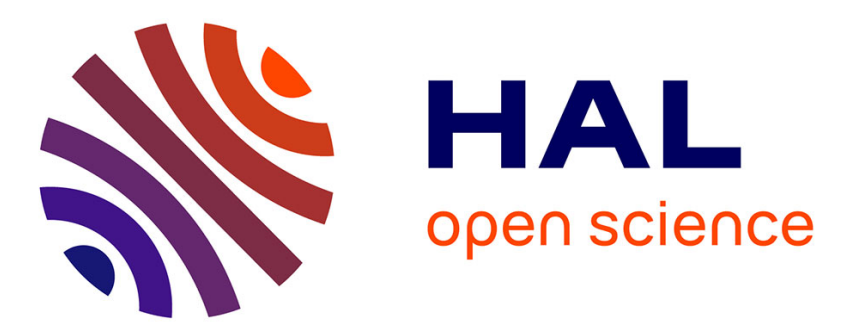

\title{
L'aube inquiétante du siècle: faillite des modèles, faiblesse des alertes
}

\author{
Alain Bauer, Philippe Baumard
}

\section{To cite this version:}

Alain Bauer, Philippe Baumard. L'aube inquiétante du siècle: faillite des modèles, faiblesse des alertes. Sécurité globale, 2011, 16. hal-03229134

\section{HAL Id: hal-03229134 \\ https://hal.science/hal-03229134}

Submitted on 18 May 2021

HAL is a multi-disciplinary open access archive for the deposit and dissemination of scientific research documents, whether they are published or not. The documents may come from teaching and research institutions in France or abroad, or from public or private research centers.
L'archive ouverte pluridisciplinaire HAL, est destinée au dépôt et à la diffusion de documents scientifiques de niveau recherche, publiés ou non, émanant des établissements d'enseignement et de recherche français ou étrangers, des laboratoires publics ou privés. 


\title{
L'aube inquiétante du siècle : faillite des modèles, faiblesse des alertes
}

\author{
Alain BAUER \\ Président du CSFRS. \\ Philippe BAUMARD \\ Président du conseil scientifique du CSFRS.
}

Le XXIe siècle semble devenu celui des crises globales. La nature nous rappelle ainsi la réalité de notre interdépendance humaine. Trois " crises " majeures hantent cette première décennie du siècle: une crise des systèmes de gouvernement; une crise de l'écosystème climatique et naturel; une crise de l'analyse et de l'anticipation.

Aucune de ces crises n'a manqué de signes avant-coureurs. Certains étaient presque imperceptibles. Beaucoup furent ignorés, ou minimisés par cette volonté collective de ne pas voir, ou de ne plus voir. Lorsque des voix s'élèvent pour avertir du mal-être global, certains ne voient que l'agitation d'un petit nombre, personne n'anticipe le soulèvement réel du pourtour méditerranéen.

Quand Olivier Roy écrit sa note à l'intention du ministre des Affaires étrangères le 24 février 2005, il ne se doute pas que le futur sera " écrit " comme il l'a déroulé: faillite des régimes autoritaires de type "baas ", popularité du principe de démocratie, transformation ou habillage nationalistes des mouvements radicaux, et un " message 
de la démocratisation qui est en train de passer ". Seul le temps de réalisation de la prédiction nuira à sa précision technique.

Dans ces crises du XXI siècle, local et global s'entremêlent. Aucune situation politique n'est équivalente : chaque côte, chaque région, chaque vallée du pourtour méditerranéen, a sa propre culture, mais il existerait aussi un " nouvel ordre " supérieur, une organisation mondiale du commerce, de la vie sociale, du consumérisme, véloces, prompts à organiser et à désorganiser, dont les flux se transmettent par les réseaux sociaux, où chaque réalité locale peut être plus ou moins amèrement comparée, dans l'instant, à tout autre. Les messages passent. La navigation cryptée et sécurisée sur Internet, autrefois apanage des États, est accessible à tous ; même au cœur des régimes autoritaires qui essayent bien d'en bloquer les ports numériques, les accès, les serveurs. Une génération usée s'accroche à son modèle du monde " multidomestique " et se croit, se veut mal informée, et croit, veut le monde mal informé. Pour essayer de passer cette frontière invisible, ce " mur " qui sépare globalement les riches et les pauvres; qui a balayé en une décennie la notion de "Nord-Sud ", chacun essaie de le contourner. Car le mur est partout, au sein de chaque société, au sein de chaque vallée, au sein de chaque quartier: il s'affiche, il est palpable à Saint-Denis, à Tunis, à Jérusalem, à Detroit. Ces crises, en gestation, en devenir, sont des crises des systèmes politiques, au sens littéral du terme: des crises de l'agir, du déséquilibre entre des strates de société de plus en plus physiquement proches, de plus en plus socialement éloignées.

On parle alors timidement d'hybridation. Des tissus sociaux criminogènes et une société prompte à se soulever se seraient rencontrés. L’Afghanistan, une fois le recul pris de trente années d'échecs, serait peut-être aussi le mélange d'aspiration légitime à la liberté et d'une terreur fanatique 0 n cherche à mettre les phénomènes dans des " cases " qui nous sont familières: là on voit une criminalité organisée et rampante, ici on voit une volonté de puissance des pays émergents; mais ce que l'on ne voit plus, c'est le phénomène le plus évident: à quel point nos modèles stratégiques, nos certitudes doctrinaires, sur le monde et ses blocs, sur notre scepticisme climatique, sur notre cartésianisme énergétique, ont volé en éclats.

En dix ans, nous avons ainsi vécu déjà trois épisodes qui, malgré l'éloignement géographique de leurs épicentres et leurs dissemblances apparentes, entretiennent en réalité des similitudes profondes. Ces trois crises signent à la fois l'échec des modèles stratégiques traditionnels et de leurs systèmes d'alertes.

La première grande crise a été terroriste avec les attentats du 11 septembre 2001 aux États-Unis. La surprise stratégique fut complète. Se croyant à l'abri derrière sa « ligne Maginot technologique ", longtemps restée aveugle à la menace du salafisme, incapable de comprendre les diverses alertes reçues, l'Amérique subit puis réagit en menant une longue et imprécise "guerre au terrorisme ". La réaction est brutale - deux longues 
guerres; des atteintes aux libertés publiques aux États-Unis et à l'étranger, pour un résultat plus que décevant...

La deuxième crise, de nature financière, surgit aussi aux États-Unis. Née de l'éclatement de la bulle immobilière (prêts hypothécaires subprimes), elle se propage au plan mondial tel un virus via la titrisation. On sait alors combien peut être toxique le mécanisme qui consiste à faire peser un risque majeur sur le prochain maillon de la chaîne; mais personne dans la communauté financière, dans la communauté "initiée ", ne réagit, même lorsque des fonds, puis des pays entiers ont généré un système de cavalerie violant les règles comptables dans un climat de compromission. Malgré sa proximité et ses conséquences durables (sociales, économiques, géopolitiques), la crise des subprimes semble déjà avoir été oubliée, comme absorbée par une amnésie collective. En attendant de feindre de découvrir la suivante, celle des dettes souveraines.

La troisième grande crise, de nature industrielle et énergétique, apparue à Fukushima (Japon), est la conséquence directe d'un tremblement de terre puis d'un tsunami qui, après avoir frappé durement un peuple, interroge désormais l'avenir de l'énergie nucléaire au plan mondial. Il existe aussi un "Olivier Roy " de la crise japonaise. Ils étaient même deux. Un sismologue, Yukinobu Okamura, qui dès 2007 avertit les autorités que la centrale de Fukushima se situe exactement à l'emplacement qui fut dévasté par le grand tsunami de l'an 869 ; puis ce fut le professeur Ishibashi Katsuhiko de l'université de Kobé, qui avertit précisément les autorités sur la faiblesse de ces digues, trop basses; comme l'avait fait le professeur Robert Bea de l'université de Californie, Berkeley à propos des digues qui entouraient la Nouvelle-Orléans en 2004. Ces crises de l'écosystème climatique sont également anthropocentriques: elles manifestent l'incapacité collective $a$ écouter, à prendre en compte les éléments de connaissance dans un monde submergé par les alertes, l'information et les signaux.

\section{Des tragédies au-delà des catastrophes}

Comment trois événements majeurs, financier, social, climatico-énergétique, ont-ils pu déclencher un phénomène si destructeur sur le plan global? Quelles dérives ont pu provoquer des situations aussi chaotiques au sens strict du terme? Comment les tensions inhérentes à ces flux cruciaux ont-elles pu se transformer en tragédies? Car il s'agit bien dans leurs causalités multiples et complexes de tragédies, pas seulement de catastrophes.

11 septembre 2001. L'Occident a permis le développement des Salafistes qui allaient ensuite se retourner contre lui. Obnubilés par la menace soviétique en Afghanistan et par la République islamique d'Iran, les États-Unis ne comprennent que tardivement 
comment leur allié d'un jour (Oussama ben Laden et le nébuleux Al-Qaïda) était devenu leur pire ennemi.

Subprimes. Les financiers américains à l'origine des subprimes n'ont eu de cesse d'user du vocabulaire catastrophique (tsunami, tempête, etc.) afın de dégager leur responsabilité individuelle. Le "système ", entité abstraite et désincarnée, avait "dysfonctionné ", selon eux, pas les hommes en responsabilité. Ces financiers préfèrent imposer une représentation fataliste des événements se dégageant ainsi opportunément de toute mise en cause personnelle et remise en question radicale.

Fukushima. Au Japon, l'action de la nature n'explique pas tout. L'ampleur de la catastrophe naturelle se comprend par l'action des hommes, en l'occurrence par une certaine dose d'aveuglement. Des décisions humaines ont bien présidé à l'implantation de cette centrale nucléaire selon des modalités spécifiques négligeant les alertes.

\section{Modèles froids, alertes inaudibles}

Il faut donc revenir à l'essentiel: la double question des modèles de risques et des alertes (gouvernementales, intellectuelles, administratives, etc.). Or l'autopsie des crises récentes conduit à un constat de défaillance majeure.

En fait, les modèles d'anticipation des risques se sont révélés trop technocratiques, trop froids. Cette froideur a conduit à chaque fois à des prises de risques manifestement excessives. Un fallacieux sentiment de sécurité a non seulement endormi la vigilance générale mais a aussi incité à développer des options dangereuses. La probabilité a envahi le raisonnement dans nombre de théories du risque. Les machines pèsent de plus en plus dans le processus de décision: en 2010, les communications machine-à-machine ont pour la première fois dépassé les communications humaines. Elles représentent aujourd'hui $60 \%$ en volume du trafic mondial de communications. Nous vivons au sein de systèmes complexes, techniques, financiers, énergétiques, très fortement dépendants de données autonomes. Les logiciels qui gèrent les prises de positions automatiques sur les marchés financiers ont des plages de "décision " inférieures à la seconde. Cette vélocité numérique est bien sûr vitale à l'économie, mais elle s'opère dans un temps humainement trop court pour permettre la " chaleur " d'un jugement critique.

Où est passée la " main visible " des managers d'Alfred Chandler? Ni la main invisible des marchés, ni la " main froide " des systèmes de gouvernance rationnels, n'ont pu éviter les crises majeures qui ont égrené ce début de XXIe siècle. Au fur et à mesure que la responsabilité de la dérive était repoussée en cascade à la périphérie du système, une doxa simple s'était imposée: les risques nés des produits hypothécaires sont raisonnables et les marchés, quoi qu'il arrive, sont omniscients et autorégulateurs : la finance innovante est 
sans danger et la " main invisible " des marchés infaillible. L'alignement idéologique et la conformité aux préjugés dominants allaient conduire à un aveuglement quasi généralisé. Les agences de notation, au cœur de conflits d'intérêts majeurs, régulatrices de fait du marché financier, distribuaient ainsi des notes de manière louangeuse à des produits financiers adossés à des prêts subprimes se révélant, pour la plupart, manipulés.

La froideur excessive des modèles de risques utilisés par la communauté financière ne peut à elle seule expliquer l'accumulation d'erreurs. La question centrale est bien la déflection, le déport des responsabilités vers les clients, l'incitation à la prise excessive de risques, ce que les Anglo-Saxons désignent comme "l'aléa moral ". Qu'elles soient tournées en de froides combinatoires probabilistes, ou qu'elles soient accueillies par de simples fins de non recevoir, comme celles reçues par Robert Bea, Olivier Roy, ou Yukinobu Okamura, les prédictions de défaillance ne sont plus entendues. Cynisme? Dilution des autorités de gouvernance? Crise de la décision?

La dérive d'une partie importante de la finance internationale peut sans doute s'expliquer par son opacité systémique; mais dans cette chaîne de décision, où se situe la véritable ignorance? Où se situe la complaisance informée? Un ménage qui finance son excès de consommation de biens électroniques sur l'hypothèque de retours immobiliers, lorsqu'il présente déjà un taux d'endettement supérieur à $70 \%$ est-il candide, ignorant ou complice? Une société de crédit connaissant une telle situation, et proposant une escalade de l'engagement dans la logique hypothécaire est-elle habile, malveillante ou criminelle? Une société du " pas vu, pas pris " est-elle tolérante, aveugle ou corrompue?

\section{L'aveuglement stratégique}

Pour les subprimes comme pour Fukushima, nous disposons déjà de toute une chronique des signaux incompris et des symptômes précurseurs négligés. Ces alertes sont restées inaudibles car noyées dans un concert d'unanimisme et de conformisme. L'examen des crises majeures, qu'elles soient d'origines terroriste, financière, industrielle, énergétique, finit toujours par montrer que le renseignement et l'analyse prédictive étaient disponibles. Elles sont simplement ignorées sur l'instant puis exhumées a posteriori. La question est donc de comprendre pourquoi ces alertes sont restées inaudibles. Les whistle blowers et autres lanceurs d'alertes existent à chaque fois mais leur voix semble se perdre dans le vacarme ambiant. Les crises contemporaines ne sont donc pas stricto sensu imprévisibles, fruits d'un manque de connaissances. Ces crises sont plutôt incompréhensibles au sens strict du terme: elles résultent d'une difficulté à combiner et mettre en relation des faits épars et en apparence autonomes.

Le déni du réel s'opère en général de bonne foi, c'est-à-dire par simple erreur ou ignorance. Il existe en la matière une littérature dense et féconde qui d'Edgar Poe à 
Jacques Lacan en passant par Sigmund Freud ou Clément Rosset décrit les différents mécanismes de l'évitement dont les individus comme les institutions sont régulièrement les jouets. Nous nous canalisons spontanément vers les informations validant nos attentes et nous repoussons avec le même empressement celles nous dérangeant profondément. Nous avons tendance à refouler toutes les réalités insupportables. Afın de surmonter ces filtres perceptuels, il est indispensable de s'interroger en permanence sur ce qui fonde nos croyances et nos préjugés. On ne peut en effet déceler ce qui est " stratégique " qu'après avoir pris conscience des nombreux biais cognitifs obstruant notre vision du monde.

Cependant, le déni du réel peut aussi puiser dans des motivations conscientes. On s'approche alors des mobiles intéressés, ceux de la dissimulation et de la fraude s'expliquant souvent par la quête du profit à court terme.

\section{Culture du raccourci, cultures arrogantes?}

Les autorités ont bien entendu les alertes. Le gouverneur de Louisiane en 2004 avait bien compris le problème des digues. Ce n'était pas seulement le prix. C'était en Louisiane la remise en cause de la compétence juridictionnelle de l'Army Corps of Engineers, un corps qui ne pouvait accepter ce diagnostic; une institution qui ne pouvait entendre des "Cassandre " ne provenant pas de ses rangs. Ce sont, dans le rapport sur l'incident de la navette Challenger, les gestionnaires du programme spatial qui ne veulent pas porter crédit aux ingénieurs du tarmac. C'est au Japon des dirigeants qui hésitent avant d'accepter l'aide technique internationale.

Cette culture de la maîtrise "froide " des risques est celle des raccourcis: raccourcis de la réalité, de la pensée stratégique, d'un système financier " robuste ", où ne sont plus jamais discutées les prémisses, que tout un chacun a oublié - a voulu oublier - pour que le raccourci remplisse sa fonction d'accélération de la décision: capitalistique, politique, et humaine. Une culture où l'on ne débat plus de sa propre culture est soit une culture infirme soit une culture arrogante.

Ces arrogances sont multiples. Il n'y a pas d'arrogance globale, mais des déclinaisons régionales, sectorielles, culturelles. Aux États-Unis, c'est l'arrogance des agences de notation, qui alertées du manque "d'orthodoxie " de leurs mélanges expérimentaux, répondent par des notes plus sévères envers les messagers. C'est une forme subtile de subversion : elle arbore les habits de la bienséance, elle déploie les façades de l'honorabilité, elle fréquente les clubs les plus exclusifs. Ce n'est pas une collusion de la "direction commune ", mais une collusion du silence raisonnable, de la "non-décision ", du respect du raccourci devenu vital. Cet orchestre d'intérêts communs, industriels et financiers aux États-Unis, nucléaires au Japon, ont à chaque fois su influencer les gouvernements et les organes de régulation à leur avantage, et ce, au détriment de l'intérêt général. À chaque 
fois, la confusion des rôles et des intérêts a produit une culture de l'arrogance (" nous savons mieux ") et de la dissimulation (" nous savons pour vous "), où la dimension frauduleuse n'est jamais explicite, rarement délibérée, mais souvent présente.

Les crises survenues ont fait de ces États initialement défaillants dans leur mission de régulation, les ultimes sauveurs des conséquences de leurs propres négligences. L'inconséquence n'est pas celle de l'industriel, ou de l'État, ou de l'ingénieur qui soudainement se voit dépeint comme un coupable intérimaire; elle devient un produit collectif, une brique générique, un " effet secondaire ".

Jusqu'à présent les États ont présenté une surface financière et logistique suffisante pour juguler les crises stratégiques successives des dix dernières années. Au nom du too big to fail, les États sont devenus les garants du dernier recours de systèmes qui refusent la mise en question ou l'introspection.

Dans ce mécanisme de dilution des responsabilités, l'aveuglement stratégique de chaque composante du système s'offre à bas prix une résilience externe le temps nécessaire. La rupture survenue, elle cherche un bouc émissaire puis tente de se relancer sur un nouveau marché.

\section{Crises spectaculaires, crises silencieuses}

Ces crises, pour dangereuses qu'elles soient, font malgré tout l'objet d'un traitement - au sens médical du terme - en raison même de leur explosivité, en fait de leur visibilité médiatique. Cependant, les " crises spectaculaires " n'épuisent pas à elles seules le champ des interrogations stratégiques. Nous sommes confrontés à des " crises silencieuses", permanentes et discrètes, dont on refuse la perception, soit parce que la menace d'effondrement n'est ni immédiate, ni "visuelle ", ne pouvant faire l'objet d'aucun Vingt heures; soit parce qu'il est politiquement impossible d'imaginer ou d'évoquer un scénario dans lequel il faudrait avouer que l'on n'a aucune variable de contrôle, aucun moyen d'intervention, et aucune perspective alternative à offrir.

Les crises criminelles en cours relèvent de cette catégorie. Leur grande létalité rejoint le flot de l'anodin, l'entrefilet du faits divers, l'anecdotique télévisuel. Les armes " légères " restent une cause principale de mortalité violente ; la contrefaçon des médicaments est une des principales sources de mortalité dans l'hémisphère sud; des sociétés entières basculent dans l'économie criminelle au Mexique, en Amérique centrale, Afrique de l'Ouest... Mais les pics, les extrêmes, la violence visuelle sont parfois si banalisées, que les réalités les plus dures en deviennent silencieuses. La dissonance a rejoint une sorte de zone d'indifférence globale: alerter, déceler, écouter sont devenus les vrais actes subversifs. 
Contrairement à ce que l'on aurait pu attendre, ou espérer, la révélation permanente au monde de ses imperfections ne trouble pas sa quiétude. Il n'y a pas d'effets domino, pas d'effondrements en chaîne entraînés par Internet. Une société de la connaissance n'est pas forcément une société agissante. La dérive criminelle de pans entiers des sociétés en Amérique centrale, la criminalisation des systèmes sociaux aussi bien en Europe que dans les États du Sud, ne sont pas des signaux faibles ou méconnus. Ils appartiennent à un autre temps stratégique, en train de se dérouler, dans un territoire à la fois présent et parallèle. Le paradoxe de cette société contemporaine est d'avoir rejoint sa critique : elle est déjà l'expression d'un futur à la Philip K. Dick où l'indifférence n'est plus orchestrée, mais une matière autonome à la perception commune; une société qui a oublié que le temps stratégique redescend toujours à l'élément unitaire du hic et nunc.

L'édition 2011 de ces Assises nationales de la recherche stratégique a donc la motivante tâche d'identifier ce qui relève de l'action stratégique au cœur de ces "brouillards de guerre " clausewitziens, environnementaux, sociaux, financiers et informatiques. Les Assises sont organisées autour de quatre thématiques qui ont marqué l'année 2011, et imprimeront notre avenir stratégique : cyber-défense et cyber-sécurité ; flux stratégiques sous tensions; les modèles stratégiques en question; la durabilité, enjeu de puissance. Ce que ces ateliers questionnent, ce sont d'abord les paradigmes existants, les logiques dominantes que l'on ne remet plus en cause, ces crises silencieuses, ou passées sous silence.

\title{
Résumé
}

Les crises et autres catastrophes ne surviennent jamais de manière inattendue. Il n'y a jamais de coup de tonnerre dans un ciel bleu. Depuis le début du XXIe siècle, nous assistons ainsi à une faillite des modèles et à une faiblesse des alertes. Des modèles froids rendent inaudibles des alertes moins rares qu'il n'y paraît au premier abord. Nous devons donc apprendre à sortir de l'aveuglement afin d'éviter les prochaines "surprises stratégiques".

\begin{abstract}
Crises and disasters never arise in a unexpected way. There is never a thunderclap in a blue skye. Since the beginning of the 21th century, we attend a bankruptcy of the models and a weakness of the alerts. Cold models make inaudible less unusual alerts than it seems at first sight. Thus, we have to learn to get out of blindness to avoid the next "strategic surprises".
\end{abstract}

